\title{
Lagrange 系统中无界轨道的变分构造
}

谨以此文致杨乐院士七十华诞

\section{程崇庆 ${ }^{1} *$, 李霞 ${ }^{(2)}$}

(1) 南京大学数学系, 南京 210093 ;

(2) 苏州科技学院数理学院, 苏州 215001

E-mail: chengcq@nju.edu.cn, lixia780527@gmail.com

收稿日期: 2009-09-29；接受日期: 2009-12-30；＊通信作者

国家重点基础研究发展计划 (973 计划) (批准号: 2007CB814800)、国家自然科学基金 (批准号: 10531050) 和江苏省基础研究 (批准号: BK2008013) 资助项目

摘要 我们将证明 $\mathbb{T}^{2}$ 上通有的测地流在通有的周期势能的扰动下无界轨道的存在性. 和前期已有的 工作不同, 这里所得到的轨道不需要充分大的初始值.

关键词 正定系统 变分法 无界轨道

MSC (2000) 主题分类 $37 \mathrm{~J} 40,37 \mathrm{~J} 50$

\section{1 引言与结论}

在本文中, 我们考虑 Lagrangian

$$
L(x, \dot{x}, t)=K(x, \dot{x})-P(x, t),
$$

其中 $K, P \in C^{r}(r=3,4, \ldots, \infty)$. 设 $x \in \mathbb{T}^{2}$ 且 $K$ 关于 $\dot{q}$ 是凸的、二阶齐次的函数, 所以 $K$ 是 $\mathbb{T}^{2}$ 上 的度量. 由 Legendre 变换, 可得到与 (1.1) 等价的 Hamiltonian:

$$
H(x, y, t)=K^{t}(x, y)+P(x, t),
$$

其中 $K^{t}$ 关于 $y$ 凸且二阶齐次. 在下文中, 我们分别用 $\phi_{L}^{t}$ 和 $\Phi_{H}^{t}$ 定义由 $L$ 和 $H$ 决定的 Lagrange 和 Hamilton 流.

文献 [1] 中以变分法研究了模型 (1.1), 该文论证了具有无界速度轨道的存在性. 相似的结果在文 献 [2-4] 中以几何方法得到. 对于已有的这些工作中所得到的轨道, 我们都要求轨道速度的初始值充 分大. 大致地讲, 无穷远点是带须环面的聚点, 在无穷远点的邻域里, 不存在构造无界轨道必须逾越的 "big gap" 问题, 因此, 延伸至无穷远的转移链就能顺利构造.

在近来的研究中, 用变分法来攻克 “big gap” 问题取得了一些进展, Arnold 扩散在 a priori unstable 系统中已被证明是一种通有现象 [6,7]. 用几何方法对此进行的研究可参考文献 [8] 和 [9]. 这些方法的 使用使得我们有可能去掉对于无界轨道初始值的限制.

对于通有的度量, 我们可作以下的假设: 
H1 对于不可约的同调类 $h_{0} \in H_{1}\left(\mathbb{T}^{2}, \mathbb{Z}\right)$, 存在唯一的最短闭测地线 $\Lambda$ 使得 $[\Lambda]=h_{0}$, 在 Morse 意义下非退化. 通过选择适当的坐标, 可假设 $h_{0}=(1,0)$.

$\mathrm{H} 2$ 存在唯一的正的 A 类 Morse 测地线 $\chi_{+}$和唯一的负的 A 类 Morse 测地线 $\chi_{-}$.

在微分动力系统里, Morse 意义下的非退化是指 $\{\Lambda, \dot{\Lambda}\}$ 对于测地流而言是双曲不变曲线; $\left(\chi_{+}, \dot{\chi}_{+}\right)$, $\left(\chi_{-}, \dot{\chi}_{-}\right)$是到 $\{\Lambda, \dot{\Lambda}\}$ 的同宿轨且存在 $a_{ \pm}^{ \pm} \in \mathbb{R}$ 使得

$$
\operatorname{dist}\left(\Lambda(t), \chi_{ \pm}\left(t+a_{ \pm}^{ \pm}\right)\right) \rightarrow 0 \text {, 当 } t \rightarrow \pm \infty,
$$

且

$$
\lim _{T \rightarrow \infty}\left\{\int_{-T+a_{ \pm}^{-}}^{T+a_{ \pm}^{+}} K\left(\chi_{ \pm}(s), \dot{\chi}_{ \pm}(s)\right) d s-\int_{-T}^{T} K(\Lambda(s), \dot{\Lambda}(s)) d s\right\}>0 .
$$

定理 1.1 设定义在 (1.1) 中的 Lagrangian $L$ 满足假设 $\mathrm{H} 1$ 和 $\mathrm{H} 2$. 则存在通有集 $\mathfrak{P} \subset\left\{\|P\|_{C^{r}} \leqslant \epsilon\right\}$, $\epsilon>0$ 充分小且对于每个 $P \in \mathfrak{P}, L$ 的 Euler-Lagrange 方程存在解 $x(t)$ 满足 $\|\dot{x}(0)\| \leqslant O(\sqrt{\epsilon})$ 且当 $t \rightarrow \infty$ 时 $\|\dot{x}(t)\| \rightarrow \infty$.

在本文中, 无界轨道是沿着一列相连的局部连接轨道构造的. 为此, 我们在上同调类里构造一条 连接 $c\left(\|c\|\right.$ 充分小) 到无穷远的路径 $\Gamma:[0,1) \rightarrow H^{1}(M, \mathbb{R}), \Gamma(0)=c, t \rightarrow 1$ 时 $\|\Gamma(t)\| \rightarrow \infty$. 对于每个 $s \in[0,1)$, 当 $\left|s-s^{\prime}\right|$ 充分小时, 存在连接 $\tilde{\mathcal{A}}(\Gamma(s))$ 到 $\tilde{\mathcal{A}}\left(\Gamma\left(s^{\prime}\right)\right)$ 个的局部连接轨道.

全局连接轨道是在局部连接轨道的基础上构建的, 为此, 在生成极小同宿轨道曲线上我们需要添 加某种极小性. 这种极小性可看作是一个 Aubry 集的“稳定流形” 和另一个 Aubry 集的 “不稳定流 形”横截相交的变分. 本文的关键点在于检验条件的通有性.

\section{2 法向双曲不变柱面}

对于 Lagrange 流 $\phi_{K}^{t}$, 存在不变柱面 $\sigma_{0} \subset T \mathbb{T}^{2}$, 它由一族不变曲线

$$
\Sigma_{0}=\{(\Lambda(s), \lambda \dot{\Lambda}(s)): \lambda \in \mathbb{R}, s \in[0,1]\}
$$

组成. 在由 $K$ 决定的 Legendre 变换 $\mathscr{L}_{K}: T \mathbb{T}^{2} \rightarrow T^{*} \mathbb{T}^{2}$ 下, 它的像是相空间中的柱面

$$
\Sigma_{0}^{*}=\mathscr{L}_{K} \Sigma_{0},
$$

该柱面关于 $\Phi_{K^{t}}$ 不变且是法向双曲的.

命题 2.1 在势能 $K$ 的小扰动下 $K \rightarrow L=K-P$, 存在关于 $\left.\phi_{L}^{t}\right|_{t=1}$ 的法向双曲不变柱面 $\Sigma$, 它 是 $\Sigma_{0}$ 的微小形变.

证明 对于 $\Sigma_{0}$ 上的未扰动的流, 每条轨道的 Lyapunov 指数是 0 , 因此, 我们可运用关于法向双 曲不变流形的基本定理 ${ }^{[10]}$. 给定充分大的 $B>0$, 存在 $D>0$ 和 $\epsilon>0$ 使得当 $P \in C^{r}\left(\mathbb{T}^{2} \times \mathbb{T}, \mathbb{R}\right)$ 且 $\|P\|_{C^{r}} \leqslant \epsilon$ 时, Lagrange 流的时间 1- 映射存在一段 $C^{r-1}$ 不变柱面 $\Sigma^{D} \subset \mathbb{T}^{2} \times\{\|\dot{x}\| \leqslant D\}$. 由于 $L$ 关 于 $\dot{x}$ 是二阶的, $\phi_{L}^{t}$ 和 Lagrange 流

$$
\tilde{L}=K\left(x, \frac{d x}{d \tau}\right)-\frac{1}{\lambda^{2}} P\left(x, \frac{\tau}{\lambda}\right)
$$

是等价的, 其中时间重新参数化为 $t \rightarrow \tau=\lambda t$. 运用关于 $\phi_{\tilde{L}}$ 的法向双曲不变流形定理, 当 $1 \leqslant \lambda \leqslant D$ 时, 在 $\mathbb{T}^{2} \times\{\|\dot{x}\| \leqslant \lambda D\}$ 我们可得到更长的一段 $C^{r-1}$ 法向双曲不变柱面. 重复这个过程, 我们可得到 满足 $\dot{x}_{1}=\infty$ 和 $\dot{x}_{1}=-\infty$ 的 $C^{r-1}$ 不变柱面. 
令 $\Sigma^{*}$ 是 $\Sigma$ 的 Legendre 变换: $\Sigma^{*}=\mathscr{L}_{L} \Sigma$. 显然, $\Sigma^{*}$ 是辛子流形, 关于 Hamiltonian 流 $\Phi_{H}^{t}$ 不变. $\Sigma^{*}$ 可看作是映射 $\psi: \Sigma_{0}^{*} \rightarrow \mathbb{R}^{2} \times \mathbb{T}^{2}$ 的像,

$$
\Sigma^{*}=\left\{x_{1}, y_{1}, x_{2}\left(x_{1}, y_{1}\right), y_{2}\left(x_{1}, y_{1}\right)\right\}
$$

该映射导致了 $\Sigma^{*}$ 上的 $2-$ 形式 $\psi^{*} \omega$,

$$
\psi^{*} \omega=\left(1+\frac{\partial\left(x_{2}, y_{2}\right)}{\partial\left(x_{1}, y_{1}\right)}\right) d x_{1} \wedge d y_{1},
$$

其中 $\omega=d x_{1} \wedge d y_{1}+d x_{2} \wedge d y_{2}$. 注意到 $\Sigma^{*}$ 上 2 阶 de Rham 上同调群的平凡性, 由 Moser 关于辛形 式同痕的讨论 ${ }^{[11]}$, 我们可得到存在 $\mathbb{T} \times \mathbb{R}$ 上的微分同胚 $\psi_{1}$ 使得

$$
\left(\psi \circ \psi_{1}\right)^{*} \omega=d x_{1} \wedge d y_{1}
$$

令 $\Phi_{H}=\left.\Phi_{H}^{t}\right|_{t=1}$. 已知 $\Sigma^{*}$ 关于 $\Phi_{H}$ 和 $\Phi_{H}^{*} \omega=\omega$ 不变, 可得到

$$
\left(\left(\psi \circ \psi_{1}\right)^{-1} \circ \Phi_{H} \circ\left(\psi \circ \psi_{1}\right)\right)^{*} d x_{1} \wedge d y_{1}=d x_{1} \wedge d y_{1},
$$

即 $\left(\psi \circ \psi_{1}\right)^{-1} \circ \Phi \circ\left(\psi \circ \psi_{1}\right)$ 保持了标准面积. 因为 $\Phi_{K^{t}}$ 是可积的且在柱面上是扭转的, 所以作为其小扰 动的 $\Phi_{H}$ 是恰当的、扭转的. 因此, 我们说在 $\Sigma^{*}$ 上的 $\Phi_{H}$ 是保面积且扭转的. 若 $r>4$, 则存在许多同 伦非平凡曲线, 包括 $\mathrm{KAM}$ 曲线. 所有的这些曲线至少是 Lipschitz 的. 给定 $\rho \in \mathbb{R}$, 存在旋转数为 $\rho$ 的 Aubry-Mather 集, 当 $\rho \in \mathbb{R} \backslash \mathbb{Q}$ 时, Aubry-Mather 集是不变圈或 Denjoy 集; 当 $\rho \in \mathbb{Q}$ 时, Aubry-Mather 集是周期轨. 在通有条件的保障下, 我们可假设对于 $\left.\Phi_{H}\right|_{\Sigma^{*}}$, 旋转数为有理数时, 不存在同伦非平凡的 曲线, 且对于有理旋转数在 $\Sigma$ 上仅有唯一的极小周期轨.

\section{3 通有转移链}

令 $\tilde{\Sigma}^{*}=\bigcup_{t \in \mathbb{T}}\left(\Phi_{H}^{t} \Sigma^{*}, t\right), \tilde{\Sigma}=\bigcup_{t \in \mathbb{T}}\left(\Phi_{L}^{t} \Sigma, t\right)$. 在这两个集合中, 存在由 $\mathscr{L}_{L}:(x, \dot{x}, t) \rightarrow(x, y, t)$ 决定 的 1-1 对应. 定义 $\tilde{\mathcal{M}}_{\rho, 0}^{*} \subset \Sigma^{*}$ 是柱面上的 Aubry-Mather 集 (不变曲线、Denjoy 集或极小周期点), 它 唯一对应到 $\tilde{\mathcal{M}}_{c}=\mathscr{L}_{L}^{-1} \bigcup_{t \in \mathbb{T}}\left(\Phi_{H}^{t} \tilde{\mathcal{M}}_{\rho, 0}^{*}, t\right) \subset \tilde{\Sigma}$. $\tilde{\mathcal{M}}_{c}$ 是 $c$ - 极小测度的支集, 即某个 $c \in H^{1}\left(\mathbb{T}^{2}, \mathbb{R}\right)$ 的 Mather 集. 扭转映射的 Aubry-Mather 集被旋转数 $\rho$ 参数化.

存在雉体 $C \subset H^{1}\left(\mathbb{T}^{2}, \mathbb{R}\right)=\mathbb{R}^{2}$ 和小的数值 $\epsilon>0$ 使得对于每个 $c \in C-O(\sqrt{\epsilon})=\{c \in C$ : $\operatorname{dist}(c, \partial C)>O(\sqrt{\epsilon})\}$, Mather 集包含于 $\tilde{\Sigma}$ 中. 其中 $\mathrm{C}$ 是顶点在原点、内部非空且包含 $\lambda(1,0)$ 的雉体. 事实上, 对于每个 $c \in C, \phi_{K}^{t}$ 的 Mather 集包含于柱面 $\tilde{\Sigma}_{0}=\bigcup_{t \in \mathbb{T}}\left(\phi_{K}^{t} \Sigma_{0}, t\right)^{[1]}$.

我们考虑路径 $\Gamma:[0,1) \rightarrow C-\delta$ 使得 $\Gamma(0) \in(C-\delta) \cap B_{2 \delta}(0)$ 且当 $s \rightarrow 1$ 时, $\|\Gamma(s)\| \rightarrow \infty$. 在这 条路径上的每个 $c$, Mañé 集 $\tilde{\mathcal{N}}(c) \subset \tilde{\Sigma}$ 且存在到 Mañé 集的一条极小同宿轨, 该极小同宿轨在下述意 义下包含于覆盖空间 $\bar{M}=\mathbb{T} \times 2 \mathbb{T}^{[7]}$

$$
\left\{\gamma_{c}(t), \dot{\gamma}_{c}(t): t \in \mathbb{R}\right\} \subset \pi_{1} \tilde{\mathcal{N}}(c, \bar{M}),
$$

其中 $\pi_{1}: \bar{M} \rightarrow M=\mathbb{T}^{2}$ 是标准投影. 因此, $\exists \delta_{c}>0$ 使得

$$
\pi_{1} \mathcal{N}(c, \bar{M}) \backslash\left(\mathcal{N}(c)+\delta_{c}\right) \neq \emptyset .
$$


其中, $A+d=\{x \in M: \operatorname{dist}(x, A) \leqslant d\}$. 我们所需论证的是这个集合的完全不连通性. 实际上, 我们 只需考虑 $\left.\tilde{\mathcal{N}}(c)\right|_{t=0}$ 为不变圈所对应的 $c$. 当 Mañé 集为 Denjoy 集和周期点时, 我们可用 $c$ - 等价去构 造连接轨道.

$c$ - 等价的定义在文献 [12] 中给出, 在文献 [6] 中证明了当 $c$ 和 $c^{\prime}$ 等价时, Aubry 集 $\tilde{\mathcal{A}}(c)$ 和 $\tilde{\mathcal{A}}\left(c^{\prime}\right)$ 可用局部极小轨道连接. 令

$$
V_{c}=\bigcap_{U}\left\{i_{U *} H_{1}(U, \mathbb{R}): U \text { 是 } \mathcal{N}_{0}(c) \text { 的邻域 }\right\} .
$$

这里, $V_{c}^{\perp}$ 是 $V_{c}$ 的零化子, 即若 $c^{\prime} \in H^{1}\left(\mathbb{T}^{n}, \mathbb{R}\right), c^{\prime} \in V_{c}^{\perp}$ 当且仅当对所有的 $h \in V_{c},\left\langle c^{\prime}, h\right\rangle=0$. 显然,

$$
V_{c}^{\perp}=\bigcup_{U}\left\{\operatorname{ker} i_{U}^{*}: U \text { 是 } \mathcal{N}_{0}(c) \text { 的邻域 }\right\} \text {. }
$$

$i_{U}: U \rightarrow M$ 是包含映射, $i_{U *}$ 和 $i_{U}^{*}$ 是由其诱导的上下同调群之间的映射. 可知存在 $\mathcal{N}(c) \cap \Sigma_{c}$ 的邻域 $U$ 使得 $V_{c}=i_{U *} H_{1}(U, \mathbb{R}), V_{c}^{\perp}=\operatorname{ker} i_{U}^{*}{ }^{[12]}$.

若存在连续曲线 $\Gamma:[0,1] \rightarrow H^{1}(M, \mathbb{R})$ 使得 $\Gamma(0)=c, \Gamma(1)=c^{\prime}$, 且对每个 $s_{0} \in[0,1]$, 存在 $\delta>0$ 使得对于任意 $s \in[0,1]$, 当 $\left|s-s_{0}\right|<\delta$ 时有 $\Gamma(s)-\Gamma\left(s_{0}\right) \in V_{\Gamma\left(s_{0}\right)}^{\perp}$, 则称 $c, c^{\prime} \in H^{1}(M, \mathbb{R})$ 是 $c$ - 等价的.

当 Mañé 集的时间 1- 截面是 Denjoy 集和周期轨时, 其同调类是平凡的, 因此与其邻近的任意上 同调类是 $c$ - 等价的.

除了通过 $c$ - 等价构造的局部连接轨道外, 还有另外一种局部连接轨道, 这种轨道类似异宿轨. 在同 宿轨与其它的同宿轨不连通的情况下, 这种异宿轨与同宿轨共存. 而对于正定系统, 当 $H_{1}\left(M, \mathcal{A}_{0}(c), \mathbb{Z}\right)$ $\neq 0$ 时, Aubry 集 $\tilde{\mathcal{A}}(c)$ 的同宿轨存在. 异宿轨的存在不是显然的, 当 Aubry 集的稳定和不稳定流形拓 扑横截相交时, 即 $\pi_{1} \mathcal{N}(c, \bar{M}) \backslash\left(\mathcal{N}(c)+\delta_{c}\right) \neq \emptyset$ 是不连通的时, 异宿轨存在.

定义 3.1 ${ }^{[7]} \quad c$ 和 $c^{\prime}$ 是 $H^{1}(M, \mathbb{R})$ 中的两个上同调类. 若存在连续曲线 $\Gamma:[0,1] \rightarrow H^{1}(M, \mathbb{R})$, $\Gamma(0)=c, \Gamma(1)=c^{\prime}$, 使得对每个 $s \in[0,1]$, 下列情形中至少有一种发生:

(I) $\Gamma(s)$ - 极小测度唯一遍历; $\pi_{1} \mathcal{N}(\Gamma(s), \bar{M}) \backslash\left(\mathcal{N}(\Gamma(s))+\delta_{s}\right)$ 完全不连通.

(II) 当 $s^{\prime}$ 和 $s$ 充分靠近时, $\Gamma(s)$ 和 $\Gamma\left(s^{\prime}\right) c$ - 等价.

此时, 称 $c$ 和 $c^{\prime}$ 以广义转移链相连.

在定义中, $c^{\prime}$ 可以是无穷远. 在这种情况下 $\Gamma$ 定义在 $[0,1)$ 上. 在本文中, 我们选择路径 $\Gamma:[0,1) \rightarrow$ $H^{1}(M, \mathbb{R})$ 使得 $\mathcal{N}(\Gamma(s), \bar{M})$ 在 $\bar{\chi}_{+}\left(\chi_{+}\right.$的提升) 的邻域内. 在下文中, 我们令 $\chi_{+}$为 $\chi$.

在我们考虑的系统里, 当旋转数是无理数时, 相关的极小测度是唯一遍历的. 旋转数是无理数时, 所对应的情形为 $c$ - 等价. 因此, 为了验证通有转移链的存在性, 我们只需证明 $\pi_{1} \mathcal{N}(\Gamma(s), \bar{M}) \backslash(\mathcal{N}(\Gamma(s))+$ $\left.\delta_{s}\right)$ 的不连通性. 为此, 我们考虑覆盖空间中的障碍函数

$$
\bar{B}_{c}^{*}(x)=\inf _{m, m^{\prime} \in \mathcal{M}_{0}(c, \bar{M})}\left\{h_{c}^{\infty}(m, x)+h_{c}^{\infty}\left(x, m^{\prime}\right)-h_{c}^{\infty}\left(m, m^{\prime}\right)\right\},
$$

$h_{c}^{\infty}$ 定义如下:

$$
h_{c}^{\infty}\left(m, m^{\prime}\right)=\liminf _{k \rightarrow \infty} \inf _{\substack{\gamma \in C^{1}([-k, k], M) \\ \gamma(-t)=m, \gamma(t)=m^{\prime}}} \int_{-k}^{k}\left(L-\eta_{c}\right)(d \gamma(s)) d s+2 k \alpha(c) .
$$

研究不连通性等价于研究 $\bar{B}_{c}^{*}$ 的极小点的完全不连通性. 


\section{4 通有性}

令 $\mathfrak{C}=\left\{c=\Gamma(s): s \in[0,1), \tilde{\mathcal{N}}_{0}(c)=\Sigma\right.$ 中的不变圈 $\}$. 本节我们要证明在通有情况下, 当 $c \in \mathfrak{C}$ 时, $\tilde{\mathcal{A}}(c)$ 的极小同宿轨与其它极小同宿轨是不连通的. 对于每条同宿轨 $d \gamma=(\gamma, \dot{\gamma})$, 可定义同调类 $[\gamma] \in H_{1}\left(M, \mathcal{A}_{0}(c), \mathbb{Z}\right) \neq 0$. 所谓的极小性是指同宿轨 $d \gamma: \mathbb{R} \rightarrow T M$ 满足: 对于任意的 $\xi,[\xi] \neq 0$, 和任 意满足 $\gamma\left(T_{i}^{+}\right) \rightarrow m^{\prime} \in A_{0}(c)$ 和 $\gamma\left(-T_{i}^{-}\right) \rightarrow m \in A_{0}(c)$ 的整数序列 $T_{i}^{ \pm} \rightarrow \infty$, 有

$$
\int_{-T_{i}^{-}}^{T_{i}^{+}}\left(L-\eta_{c}\right)(d \gamma(t), t) d t \leqslant \int_{-T_{i}^{-}}^{T_{i}^{+}}\left(L-\eta_{c}\right)(d \xi(t), t) d t .
$$

令 $\bar{\chi}=\left(\bar{\chi}_{1}, \bar{\chi}_{2}\right)$ 是 $\chi$ 在 $\bar{M}=\mathbb{T} \times 2 \mathbb{T}$ 中的提升, $\bar{\chi}_{2}\left(T_{i}^{+}\right)-\bar{\chi}_{2}\left(-T_{i}^{-}\right)>0$, 其中 $\mathbb{Z} \ni T_{i}^{ \pm} \rightarrow \infty$ 使得 $\chi\left(T_{i}^{+}\right), \chi\left(-T_{i}^{-}\right) \rightarrow m \in \Lambda$. 由假设 $\mathrm{H} 2$, 每条 $\left(\chi, \lambda_{c} \dot{\chi}\right)$ 是流 $\phi_{K}^{t}$ 的到 $\tilde{\mathcal{A}}(c)$ 的和 $[\chi]$ 是同一同调类的唯一 极小同宿轨. 在覆盖空间 $T \bar{M}$ 中, $\left(\left\{\bar{\chi}(t), \lambda_{c} \dot{\bar{\chi}}(t): t \in \mathbb{R}\right\}\right)$ 是 Lipschitz 图.

定义 $\Sigma_{0,1}$ 和 $\Sigma_{0,2}$ 是 $\Sigma_{0}$ 的提升, $\Sigma_{0,1}$ 包含了 $\left(\bar{\chi}, \lambda_{c} \dot{\bar{\chi}}\right)$ 的 $\alpha$ - 极限集, $\Sigma_{0,2}$ 包含了 $\left(\bar{\chi}, \lambda_{c} \dot{\bar{\chi}}\right)$ 的 $\omega$ 极限集. 在小扰动 $K \rightarrow L=K-P$ 下, $\phi_{L}$ 的不变柱面有两个提升 $\Sigma_{1}$ 和 $\Sigma_{2}, \Sigma_{i}$ 在 $\Sigma_{0, i}$ 的小邻域内 $(i=1,2)$. 每条不变曲线 $\Upsilon_{c}$ 也有两个提升 $\Upsilon_{c, i} \subset \Sigma_{i}(i=1,2)$.

$\Sigma$ 关于 $\phi_{L}$ 是法向双曲的, 其上的每一点有稳定和不稳定纤维. 考虑 $\Sigma_{1}$ 中不变曲线 $\Upsilon_{c, 1}$ 的不稳 定流形 $W_{c, 1}^{u}$ 和 $\Upsilon_{c, 2}$ 的稳定流形 $W_{c, 2}^{s}$. 由于沿整条曲线 $\left(\bar{\chi}, \lambda_{c} \dot{\bar{\chi}}\right)$ 保持水平, $\phi_{L}$ 是 $\phi_{K}$ 的小扰动, $W_{c, 1}^{u}$ 在 $\left\{\left(\bar{\chi}(t), \lambda_{c} \dot{\bar{\chi}}(t)\right): t \in(-\infty, T)\right\}$ 的一个邻域内保持水平, 该邻域从 $\Sigma_{1}$ 延伸至 $\Sigma_{2}$ 的 $a$ - 邻域, 记为 $\Sigma_{2}+a, a>0$ 是一个小的数值. 同样的, $W_{c, 1}^{s}$ 在 $\left\{\left(\bar{\chi}(t), \lambda_{c} \dot{\bar{\chi}}(t)\right): t \in(-T, \infty)\right\}$ 的一个邻域内保持水平, 该邻域从 $\Sigma_{2}$ 延伸至 $\Sigma_{1}+a$. 因此, 存在开集 $U \subset \bar{M} \backslash\left(\left(\pi \Sigma_{1}+a\right) \cup\left(\pi \Sigma_{2}+a\right)\right)$ 包含 $\bar{\chi}$ 的一段, 在 $U$ 上 $W_{c, 1}^{u}$ 和 $W_{c, 2}^{s}$ 都保持水平, $\pi$ 是指沿切丛的投影: $T M \rightarrow M$. 因此, 对于每个 $x \in U$ 和每个 $c \in \mathfrak{C}$, 存在 唯一的向前的 $c$ - 极小曲线 $\bar{\gamma}_{c}^{+}$和向后的 $c$ - 极小曲线 $\bar{\gamma}_{c}^{-}$使得 $\alpha\left(d \bar{\gamma}_{c}^{-}\right) \subset \tilde{\Sigma}_{1}, \omega\left(d \bar{\gamma}_{c}^{+}\right) \subset \tilde{\Sigma}_{2}$.

在 Legendre 变换 $\mathscr{L}_{L}$ 下, $W_{c, 1}^{u}$ 和 $W_{c, 2}^{s}$ 的像在 $U$ 上保持水平. 因此, 存在生成函数 $u_{c}^{-}, u_{c}^{+} \in$ $C^{1,1}(\bar{M}, \mathbb{R})$ 使得 $\mathscr{L}_{L}\left(\left.W_{c, 1}^{u}\right|_{U}\right)=\left.d u_{c}^{-}\right|_{U}, \mathscr{L}_{L}\left(\left.W_{c, 2}^{s}\right|_{U}\right)=\left.d u_{c}^{+}\right|_{U}$ 且

$$
\bar{B}_{c}^{*}=u_{c}^{-}-u_{c}^{+}-\text {常数 }
$$

(参见文献 [6]). 下面我们要说明 $\left.\bar{B}_{c}^{*}\right|_{R}$ 的极小点是完全不连通的.

考虑势能扰动 $H \rightarrow H+P_{\delta}$, 即 $P_{\delta} \in C^{r}(M \times \mathbb{T}, \mathbb{R})$. 定义 $z_{c, \delta}^{-}, z_{c, \delta}^{+}$分别是 $\Phi_{H+P_{\delta}}^{t}$ 的向前或向后 的 $c$ - 极小轨道. 因为 $\Phi_{H}^{t}$ 是 $\Phi_{K^{t}}^{t}$ 的小扰动, $z_{c, \delta}^{ \pm}$穿过 $\left\{x_{1}^{*}-d, x_{1}^{*}+d\right\}$ 的时间量级为 $O(d)$, 若它们与 $\mathscr{L}_{L}\left(\chi, \lambda_{c} \dot{\chi}\right)$ 靠近, $\lambda_{c}$ 是某个常数:

$$
\max \left\{\left|t^{\prime}-t\right|: z_{c, \delta}^{ \pm}(t), z_{c, \delta}^{ \pm}\left(t^{\prime}\right) \in\left\{x_{1}^{*}-d, x_{1}^{*}+d\right\}\right\}=O(d) .
$$

令 $\Delta z_{c}^{ \pm}=z_{c, \delta}^{ \pm}-z_{c}^{ \pm}$, 它是如下变分方程的解:

$$
\Delta \dot{z}_{c}^{ \pm}=J \frac{\partial^{2} H}{\partial z^{2}} \Delta z_{c}^{ \pm}-\left[\begin{array}{c}
0 \\
\partial_{x} P_{\delta}
\end{array}\right]+O\left(\left\|\Delta z_{c}^{ \pm}\right\|^{2}+\left\|P_{\delta}\right\|\left\|\Delta z_{c}^{ \pm}\right\|\right),
$$

相应的函数在 $\left(z_{c}^{ \pm}(t), t\right)$ 处取值. 令 $\Psi_{c}^{ \pm}(t)$ 是线性方程

$$
z=J \frac{\partial^{2} H}{\partial z^{2}} z
$$


的基本解矩阵, $\Psi_{c}^{ \pm}(0)=I, I$ 是单位矩阵. 若 $\operatorname{supp} P_{\delta} \subseteq \mathbb{T} \times\left[x_{1}^{*}-d, x_{1}^{*}+d\right] \times \mathbb{T}, z_{c, \delta}^{-}(0) \in\left\{x_{1}=x_{1}^{*}-d\right\}$, 则 $\Delta z_{c}^{-}(0)=0$ 且

$$
\left[\begin{array}{c}
\Delta x_{c}^{-} \\
\Delta y_{c}^{-}
\end{array}\right]=-\Psi_{c}^{-}(t) \int_{0}^{t}\left(\Psi_{c}^{-}\right)^{-1}\left[\begin{array}{c}
0 \\
\partial P_{\delta}
\end{array}\right] d s+O\left(\left\|P_{\delta}\right\|^{2}\right),
$$

积分中的函数在 $\left(z_{c}^{-}(s), s\right)$ 取值. 因为 $\Psi_{c}^{-}(0)$ 是单位矩阵且极小曲线穿越 $\mathbb{T} \times\left[x_{2}^{*}-d, x_{2}^{*}+d\right]$ 的时间 阶为 $O(d)$, 所以有

$$
\Psi_{c}^{-}(t)=I+O(d), \quad \forall t \in[0, O(d)] .
$$

因此, 在 $\mathbb{T} \times\left[x_{1}^{*}-d, x_{1}^{*}+d\right] \times[0, O(d)]$ 中, 下列式子成立:

$$
\begin{aligned}
& \Delta x_{c}^{-}(t)=O\left(d^{2}\left\|P_{\delta}\right\|\right)+O\left(d\left\|P_{\delta}\right\|^{2}\right), \\
& \Delta y_{c}^{-}(t)=-\int_{0}^{t} \partial_{x} P_{\delta} d s+O\left(d^{2}\left\|P_{\delta}\right\|\right)+O\left(d\left\|P_{\delta}\right\|^{2}\right) .
\end{aligned}
$$

类似的, 在条件 $z_{c, \delta}^{+}(0) \in\left\{x_{1}=x_{1}^{*}+d\right\}, \Delta z_{c}^{+}(0)=0$ 保证下, 在 $\mathbb{T} \times\left[x_{1}^{*}-d, x_{1}^{*}+d\right] \times[-O(d), 0]$ 中, 下 列式子成立:

$$
\begin{aligned}
& \Delta x_{c}^{+}(t)=O\left(d^{2}\left\|P_{\delta}\right\|\right)+O\left(d\left\|P_{\delta}\right\|^{2}\right), \\
& \Delta y_{c}^{+}(t)=\int_{t}^{0} \partial_{x} P_{\delta} d s+O\left(d^{2}\left\|P_{\delta}\right\|\right)+O\left(d\left\|P_{\delta}\right\|^{2}\right),
\end{aligned}
$$

积分中的函数在 $\left(z_{c}^{+}(s), s\right)$ 上取值.

对于每个 $c \in \mathfrak{C}$ 、时间 $\tau$ 和与 $\chi$ 靠近的每点 $x \in \mathbb{T} \times\left[x_{1}^{*}-d, x_{1}^{*}+d\right]$, 存在唯一决定的两点 $x_{c}^{-} \in\left\{x_{1}=x_{1}^{*}-d\right\}, x_{c}^{+} \in\left\{x_{1}=x_{1}^{*}+d\right\}$ 和唯一决定的时间 $\tau_{c}^{-}, \tau_{c}^{+}$使得初始值为 $\left(x_{c}^{-}, \tau_{c}^{-}\right)$的负向 $c-$ 半静态曲线 $x_{c}^{-}(t)$ 在时间 $\tau-\tau_{c}^{-} \leqslant O(d)$ 内到达该点; 初始值为 $\left(x_{c}^{+}, \tau_{c}^{+}\right)$的正向 $c$ - 半静态曲线 $x_{c}^{+}(t)$ 在时间 $\tau_{c}^{+}-\tau \leqslant O(d)$ 内到达该点. 注意到 $y=\partial_{x} u$, 由 (4.1) 的表达式, 可得到

$$
\partial_{x} \Delta \bar{B}_{c, P_{\delta}}^{*}(x, \tau)=-\left(\int_{\tau_{c}-}^{\tau} \partial_{x} P_{\delta} d s+\int_{\tau}^{\tau_{c}^{+}} \partial_{x} P_{\delta} d s\right)+O\left(d^{2}\left\|P_{\delta}\right\|+d\left\|P_{\delta}\right\|^{2}\right),
$$

其中 $\Delta \bar{B}_{c, P_{\delta}}^{*}=\bar{B}_{c, P_{\delta}}^{*}-\bar{B}_{c}^{*}, \bar{B}_{c, P_{\delta}}^{*}$ 是扰动后的 Lagrangian 在 $c$ 处的障碍函数. 第一个积分沿 $x_{c}^{-}$计算, 第二个积分沿 $x_{c}^{+}$计算. 显然, 障碍函数在 $\chi$ 的小邻域内是光滑的.

对于我们所考虑的问题, $P_{\delta}$ 是关于 $\left(x_{1}, t\right)$ 的函数, $\operatorname{supp} P_{\delta}=\left[x_{1}^{*}-d, x_{1}^{*}+d\right] \times \mathbb{T}$, 因此,

$$
\begin{aligned}
& \partial_{x_{1}} \Delta \bar{B}_{c, P_{\delta}}^{*}(x, \tau)=-\left(\int_{\tau_{c}-}^{\tau} \partial_{x_{1}} P_{\delta} d s+\int_{\tau}^{\tau_{c}^{+}} \partial_{x_{1}} P_{\delta} d s\right)+O\left(d^{2}\left\|P_{\delta}\right\|+d\left\|P_{\delta}\right\|^{2}\right), \\
& \partial_{x_{2}} \Delta \bar{B}_{c, P_{\delta}}^{*}(x, \tau)=O\left(d^{2}\left\|P_{\delta}\right\|+d\left\|P_{\delta}\right\|^{2}\right) .
\end{aligned}
$$

因为 $L$ 是 $K$ 的小扰动, 对于每个 $c \in \mathfrak{C}$, 每条极小同宿曲线在 $\chi$ 的 $\epsilon$ - 邻域内. 因此, 我们只需考虑在 $\chi+O(\epsilon) \cap\left[x_{1}^{*}-d, x_{1}^{*}+d\right] \times \mathbb{T}$ 里的障碍函数, $\exists \lambda_{c}, t, t^{\prime} \in \mathbb{R}$ 使得

$$
\begin{aligned}
& \left|\chi\left(\lambda_{c} s\right)-\gamma_{c}^{+}(s+t)\right| \leqslant O(\epsilon), \quad \forall s \geqslant 0, \\
& \left|\chi\left(\lambda_{c} s\right)-\gamma_{c}^{-}\left(s+t^{\prime}\right)\right| \leqslant O(\epsilon), \quad \forall s \leqslant 0 .
\end{aligned}
$$


从而得到

$$
\begin{aligned}
& \partial_{x_{1}} \Delta \bar{B}_{c, P_{\delta}}^{*}(x, \tau)=-\frac{1}{\lambda_{c}} \int_{0}^{T_{d}} \partial_{x_{1}} P_{\delta}\left(\chi_{1}(s), \frac{s}{\lambda_{c}}+t\right) d s+O\left(d^{2}\left\|P_{\delta}\right\|+d\left\|P_{\delta}\right\|^{2}+\epsilon d\left\|P_{\delta}\right\|\right), \\
& \partial_{x_{2}} \Delta \bar{B}_{c, P_{\delta}}^{*}(x, \tau)=O\left(d^{2}\left\|P_{\delta}\right\|+d\left\|P_{\delta}\right\|^{2}\right),
\end{aligned}
$$

若我们记 $x=\left(x_{1}, x_{2}\right), \chi=\left(\chi_{1}, \chi_{2}\right)$, 则 $\chi_{1}(t)=x_{1}, T_{d}>0$ 使得 $\bar{\chi}_{1}(t)=x_{1}^{*}-d, \bar{\chi}_{1}(t)=x_{1}^{*}+d$.

在 $C^{r}\left(\left[x_{1}^{*}-d, x_{1}^{*}+d\right] \times \mathbb{T}, \mathbb{R}\right)$ 中我们定义一个等价关系: 若存在 $\lambda, \lambda^{\prime} \in \mathbb{R}$ 使得

$$
u\left(\chi(s), \frac{s}{\lambda}+t\right)=u^{\prime}\left(\chi(s), \frac{s}{\lambda^{\prime}}+t\right), \quad \forall s, t \in \mathbb{R},
$$

则称 $u, u^{\prime} \in C^{r}\left(\left[x_{1}^{*}-d, x_{1}^{*}+d\right] \times \mathbb{T}, \mathbb{R}\right)$ 是等价的, 记为 $u \sim u^{\prime}$. 对于任意 $u, u^{\prime} \in C^{r}\left(\left[x_{1}^{*}-d, x_{1}^{*}+d\right] \times \mathbb{T}, \mathbb{R}\right)$ 定义

$$
\operatorname{dist}\left(u, u^{\prime}\right)=\min _{\substack{\lambda, \lambda^{\prime} \\ s, t}}\left|u\left(\chi(s), \frac{s}{\lambda^{\prime}}+t\right)-u^{\prime}\left(\chi(s), \frac{s}{\lambda^{\prime}}+t\right)\right| .
$$

这是商空间 $C^{r}\left(\left[x_{1}^{*}-d, x_{1}^{*}+d\right] \times \mathbb{T}, \mathbb{R}\right) / \sim$ 上的一个度量. 显然, 在此度量下, $C^{r}\left(\left[x_{1}^{*}-d, x_{1}^{*}+d\right] \times \mathbb{T}, \mathbb{R}\right) / \sim$ 是无穷维的. 令 $\mathfrak{B}_{\delta}$ 是 $C^{r}\left(\left[x_{1}^{*}-d, x_{1}^{*}+d\right] \times \mathbb{T}, \mathbb{R}\right) / \sim$ 中以原点为圆心、半径为 $\delta$ 的球.

引理 4.1 存在小的数 $\delta>0$ 使得对于任意 $P_{\delta}, P_{\delta}^{\prime} \in \mathfrak{B}_{\delta}$, 有

$$
\max _{x, \tau}\left|\partial_{x_{1}} \Delta B_{c, P_{\delta}}^{*}-\partial_{x_{1}} \Delta B_{c^{\prime}, P_{\delta}^{\prime}}^{*}\right| \geqslant \frac{1}{2} \min \left\{\lambda_{c}^{-1}, \lambda_{c^{\prime}}^{-1}\right\} \operatorname{dist}\left(P_{\delta}, P_{\delta}^{\prime}\right) .
$$

证明 对于充分小的 $\delta>0$, 从 (4.2) 可得到.

定理 4.2 对于每个紧集 $\mathfrak{C}_{0} \subset \mathfrak{C}$, 存在开稠集 $\mathfrak{P} \subset\left\{\|P\|_{C^{r}} \leqslant \delta\right\}$ 使得对于每个 $P \in \mathfrak{P}$ 和 $c \in \mathfrak{C}_{0}$, $\phi_{L}^{t}$ 的到 $\tilde{\mathcal{A}}(c)$ 的极小同宿轨是完全不连通的.

证明 因为 $L$ 是 $K$ 的小扰动, Mañé 集包含在 $\chi$ 的小邻域里. 因此, 若 $\mathcal{Z}=\left\{x: \bar{B}_{c}^{*}(x, t)=\right.$ $\left.\min _{x} \bar{B}_{c}^{*}(x, t)\right\}$ 在 $t$ - 截面包含了连续曲线, 则其投影包含了区间, 定义该区间为 $\left[x_{1}^{*}-d, x_{1}^{*}+d\right]$.

假设稠性不成立, 则存在一个 $k$ 维球 $\mathfrak{B}_{k, \delta} \subset \mathfrak{B}_{\delta}$, 当 $P_{\delta} \in \mathfrak{B}_{k, \delta}$ 和 $c \in \mathfrak{C}_{0}$ 时, $\bar{B}_{c, P_{\delta}}^{*}$ 的极小点在每 个时间截面都包含一条连续曲线. 用 $\mathscr{B}\left(\mathfrak{B}_{k, \delta}\right)$ 表示这样的障碍函数集.

由于 $P_{\delta}$ 和 $x_{1}$ 无关, 从引理 4.1 可得

$$
\max \left|\Delta B_{c}^{*}-\Delta B_{c^{\prime}}^{*}\right| \geqslant \frac{1}{2} \min \left\{\lambda_{c}^{-1}, \lambda_{c^{\prime}}^{-1}\right\} \operatorname{dist}\left(P_{\delta}, P_{\delta}^{\prime}\right) .
$$

这说明 $\mathscr{B}\left(\mathfrak{B}_{k, \delta}\right)$ 的 box 维数不超过 $k$.

另一方面, 在文献 [6] 中已经证明了障碍函数集 $\bigcup_{c \in \mathfrak{C}_{0}} B_{c}^{*}$ 的 box 维数不超过 2 , 若选择 $k \geqslant 3$, 我 们便可得到矛盾. 开性是显然的.

为了说明通有链的存在性是一个通有的性质, 我们需要说明对于对于所有的 $c \in \mathfrak{C}$ 极小同宿轨的 完全不连通性, 因为 $\mathfrak{C}$ 是可数紧集的并, 所以这可由定理 4.2 推出.

我们也可从文献 [1] 中加在 Melnikov 函数上的假设推出此结论:

$$
M(t)=\lim _{T \rightarrow \infty}\left\{\int_{-T+a^{-}}^{T+a^{+}} U\left(\chi_{+}(s), t\right) d s-\int_{-T}^{T} U(\Lambda(s), t) d s\right\}-\left(a^{+}-a^{-}\right)\langle U(\cdot, t)\rangle_{\gamma},
$$

其中 $\langle U(\cdot, t)\rangle_{\gamma}$ 是指 $U$ 沿着最短闭测地线 $\gamma$ 的平均作用量. 文中要求 $M(t)$ 的极小点是完全不连通的, 这是一个通有的假设.

显然, 当上同调类充分靠近无穷远处时, $M(t)$ 处处不为常数意味着同宿曲线集合的不连通性. 


\section{5 变分框架}

通有转移链的存在性保证了满足下述条件的上同调类 $c_{0}, c_{1}, \ldots, c_{k}, \ldots$ 的存在性:

1) 对于每个 $c_{k}$, Mather 集包含在不变柱面上;

2) $k \rightarrow \infty$ 时, $\left\|c_{k}\right\| \rightarrow \infty$;

3) $\tilde{\mathcal{A}}\left(c_{i}\right)$ 和 $\tilde{\mathcal{A}}\left(c_{i+1}\right)$ 或者以基于 $c$ - 等价的局部极小轨道相连, 或者基于孤立同宿轨存在的局部极 小轨道相连.

选择有限多的同调类 $c_{1}, c_{2}, \ldots, c_{k}$, 文献 [7] 中的工作表明

$$
L_{k}=L-\eta_{0}-\sum_{i=0}^{k}\left(-\tau_{i}\right)^{*}\left(\mu_{i}+\psi_{i}\right)
$$

的极小化子 $\gamma_{k}: \mathbb{R} \rightarrow M$ 在一定的限制条件下是连接 $\tilde{\mathcal{M}}\left(c_{0}\right)$ 到 $\tilde{\mathcal{M}}\left(c_{k}\right)$ 的轨道. 显然, 当 $k, t \rightarrow \infty$ 时, $\left|\dot{\gamma_{k}}(t)\right| \rightarrow \infty$. 令 $k \rightarrow \infty$, 一系列极小化子 $\left\{\gamma_{k}, \gamma_{k+1}, \ldots\right\}$ 的存在意味着无界轨道的存在性, 这样完成了 定理 1.1 的证明.

\section{参考文献}

1 Mather J N. Variational construction of trajectories for time periodic Lagrangian systems on the two torus. Manuscript, 1997

2 Bolotin S, Treschev D. Unbounded growth of energy in nonautonomous Hamiltonian systems. Nonlinearity, 1999, 12: 365-388

3 Delshama A, de la Llave R, Seara T M. A geometric approach to the existence of orbits with unbounded energy in generic periodic perturbations by a potential of generic geodesic flows of $\mathbb{T}^{2}$. Comm Math Phys, 2000, 209: 353-392

4 Delshama A, de la Llave R, Seara T M. Orbits of unbounded energy in quasi-periodic perturbations of geodesic flows. Adv Math, 2006, 202: 64-188

5 Arnol'd V I. Instability of dynamical systems with several degrees of freedom. Sov Math Dokl, 1964, 5: 581-585

6 Cheng C-Q, Yan J. Existence of diffusion orbits in a priori unstable Hamiltonian systems. J Differ Geom, 2004, 67: $457-517$

7 Cheng C-Q, Yan J. Arnold diffusion in Hamiltonian systems: a priori unstable case. J Differ Geom, 2009, 82: 229-277

8 Delshams A, de la Llave R, Seara T M. Geometric mechanism for diffusion in Hamiltonian systems overcoming the large gap problem: heuristic and rigorous verification of a model. Mem Amer Math Soc, 2006, 179: 1-141

9 Treschev D V. Evolution of slow variables in a priori unstable Hamiltonian systems. Nonlinearity, 2004, 17: 1803-1841

10 Hirsch M W, Pugh C C, Shub M. Invariant Manifolds. In: Lect Notes Math, vol. 583. Berlin: Springer-Verlag, 1977

11 Moser J K. On the volume elements on a manifold. Trans Amer Math Soc, 1965, 120: 286-294

12 Mather J. Variational construction of connecting orbits. Ann Inst Fourier (Grenoble), 1993, 43: 1349-1386 